\title{
Blood Transfusion in Obstetrics
}

\author{
Nigam $A,{ }^{1}$ Prakash $A,{ }^{2}$ Saxena $P^{1}$
}

${ }^{1}$ Department of Gynecology and Obstetrics

${ }^{2}$ Department of Medicine

Lady Hardinge Medical College and Smt. Sucheta Kriplani Hospital, New Delhi

India

\section{Corresponding Author}

Aruna Nigam

Department of Gynecology and Obstetrics

Lady Hardinge Medical College and Smt. Sucheta Kriplani Hospital, New Delhi

India

E-mail: prakashanupam@hotmail.com

\section{Citation}

Nigam A, Prakash A, Saxena P. Blood Transfusion in Obstetrics. Kathmandu Univ Med J 2013;44(4):355359.

\begin{abstract}
Transfusion of blood and blood components is a common practice in obstetric wards but it is not without risk. The incidence of transfusion reactions varies from 4 in every hundred transfusions for non-haemolytic reactions to one in every 40,000 for haemolytic transfusion reactions. The physiological basis of blood transfusion is outlined in this article. Most of the donated blood is processed into components: packed red cells (PRBCs), platelets, and fresh frozen plasma (FFP) or cryoprecipitate. Various alternatives to blood transfusion exist and include autotransfusion, pre-autologous blood storage, use of oxygen carrying blood substitutes and intraoperative cell salvage. Despite the risks associated with transfusions, obstetricians are frequently too aggressive in transfusing blood and blood products to their patients. Acute blood loss in obstetrics is usually due to placenta praevia, postpartum blood loss and surgery related. An early involvement of a consultant obstetrician, anaesthetist, haematologist and the blood bank is essential. There are no established criteria for initiating red cell transfusions and the decision is purely based on clinical and haematological parameters, which have been discussed along with the general principles of blood transfusion in obstetrics and some practical guidelines.
\end{abstract}

\section{KEYWORDS}

Cryoprecipitate, fresh frozen plasma, packed cells, platelets

\section{INTRODUCTION}

Transfusion of blood and blood components is a common practice in obstetric wards. Moreover, obstetric conditions associated with the need for blood transfusion are associated with considerable morbidity and mortality, if not properly managed. Obstetric haemorrhage continues to be a major cause of maternal mortality in India with 'substandard management' being a contributor in $80 \%$ of the cases. ${ }^{1}$ High risk pregnancies with an anticipated loss greater than $1000 \mathrm{ml}$ should be strongly advised to deliver in a setting where transfusion support and intensive care facilities are available.

Blood transfusion may be a life-saving procedure but it is not without risk. The incidence of transfusion reactions varies from 4 in every hundred transfusions for non haemolytic reactions to one in every 40,000 for haemolytic transfusion reactions. Although, recipients can develop transfusion-transmitted infections as well as suffer immunological sequelae such as red cell alloimmunisation; but the major risk associated with blood transfusion is of a patient receiving an 'incorrect blood component. ${ }^{1}$ Strict adherence to correct sampling, cross-match and administration procedures is most important way to avoid these complications. The present article deals with the basis of blood transfusion, its indications in routine obstetric practice and component therapy.

\section{Physiological Basis of Transfusion}

The blood and its components are transfused primarily to

- Enhance oxygen carrying capacity of blood.

- Replace clotting factors which are either lost, consumed or not produced.

Under normal circumstances the oxygen delivery to the tissues is $1000 \mathrm{ml} / \mathrm{min}$ and the oxygen consumption is $200 \mathrm{ml} / \mathrm{min}$. Hence, the ratio of oxygen delivery to oxygen consumption is $5: 1$. In patients with anaemia, hypoxia or 
myocardial failure in whom the delivery of oxygen cannot be increased and in whom the consumption is increased, this $5: 1$ ratio will fall with the patient using up the inherent oxygen reserves. This scenario persists until the ratio falls to 2:1 up to which level the patient remains stable. Thus the recommendation to transfuse a patient must focus on compensatory ability of the patient and physiologic parameters and not only on the packed cell volume (PCV) as is the case generally. Transfusion therefore, is only necessary when patients cannot compensate for their anaemia.

When the compensatory mechanisms are normal with an adequate oxygen delivery it may not be necessary to transfuse patients until the PCV drops below $16 \%$ (Hemoglobin, $\mathrm{Hb}<5.3 \mathrm{~g} / \mathrm{dL}$ ) and in patients with poor compensatory mechanisms may only be advised when the PCV drops below $25 \%(\mathrm{Hb}<8.3 \mathrm{~g} / \mathrm{dL})^{2,3}$

Release of the oxygen to tissues mainly depends on oxygen saturation of $\mathrm{Hb}$ besides other factors. As saturation increases, the decreasing affinity will cause the enhanced release of oxygen to tissues. Partial pressure of oxygen required to saturate $50 \%$ of the $\mathrm{Hb}$ molecule is called $\mathrm{P}-50$. This is increased with fever, acidosis, increased 2-3 diphosphoglycerate (DPG), thus oxygen is released to tissues with greater ease in these circumstances. However with hypothermia, alkalosis and decreased 2-3 DPG, affinity of oxygen is increased and release is decreased.

$\mathrm{Hb}$ normally ranges between $12-18 \mathrm{~g} / \mathrm{dL}$ depending on race, age, sex and medical condition. The capacity of tolerating the lower concentrations of $\mathrm{Hb}$ depends on

- the rate and magnitude of blood loss

- state of tissue perfusion

- pre-existing cardiopulmonary disease.

\section{Different Components of Blood Transfusion}

Blood components intended for blood transfusion are routinely collected as anticoagulated whole blood $(450 \mathrm{ml})$. Most of the donated blood is processed into components: packed red cells (PRBCs), platelets, and fresh frozen plasma (FFP) or cryoprecipitate. Whole blood is first separated into PRBCs and platelet rich plasma by slow centrifugation. The platelet rich plasma is then centrifuged at high speed to yield one unit of random donor (RD) platelet and one unit of FFP. Cryoprecipitate is produced by thawing FFP to precipitate the plasma proteins, and then separated by centrifugation.

Whole Blood: It is stored at $4^{\circ} \mathrm{C}$ to sustain erythrocyte viability. It is the ideal component for the patients who have sustained acute haemorrhage (blood loss $>25 \%$ of total blood volume) as it improves the oxygen carrying capacity and results in blood volume expansion.

Disadvantages of whole blood are that

- Platelet dysfunction and degradation of some clotting factors occur within 24-48 hours of storage.

- Levels of 2-3 DPG may decrease by $30 \%$ in blood stored for greater than two weeks, by $60-70 \%$ in three weeks, thus significantly diminishing the ability to release oxygen to tissues.

Packed Red Blood Cells ( PRBC) : This is prepared by removing 200 cc of plasma from fresh whole blood, to achieve a final hematocrit of $70-80 \%$. They are stored in liquid state at $4^{\circ} \mathrm{C}$ or frozen at $-80^{\circ} \mathrm{C}$. It is kept anticoagulated with CPD (citrate, phosphate, dextrose). Survival rate of blood cells decreases from $90 \%$ with immediate transfusion to $65 \%$ at six weeks of storage. Pre-storage leucocyte reduction of PRBCs is recommended universally to prevent certain adverse reactions i.e. posttransfusion fever, cytomegalovirus (CMV) infections, and alloimmunization. ${ }^{4}$ Prestorage filtration appears superior to bedside filtration as smaller amount of cytokine is generated in the stored product.

Cryopreserved RBC : This technique utilizes rapid cooling of PRBC to $-80^{\circ} \mathrm{C}$ in $40 \%$ glycerol. 2-3 DPG level remains normal. Antigenic reactions are minimum in this technique. Large quantities of red cells can be stored for many years. But high expense is a significant disadvantage.

Platelets : It is collected by repeated centrifugation of fresh whole blood, and suspension in 30-50 cc of plasma at $22^{\circ} \mathrm{C}$. It remains viable for up to five days and is most efficacious if used within 24-48 hours of pooling as they lose ability to produce thromboxane A-2, a potent vasoconstrictor and platelet aggregator gradually over five days. It should be $\mathrm{ABO}$ and $\mathrm{Rh}$ compatible, since donor plasma is present. Risk of infectious complications are proportionate to number of donors.

Apheresis technology is used for collection of multiple units of platelets from single donor. These single donor apheresis platelets (SDAP) contain the equivalent of at least 6 units of random-donor platelets and have fewer contaminating leukocytes than pooled random-donor platelets.

The threshold for the prophylactic platelet transfusion is $10,000 / \mathrm{mL}$. In patients without fever or infections, a threshold of $5000 / \mathrm{mL}$ may be sufficient to prevent spontaneous hemorrhage. For invasive procedures 50,000/ $\mathrm{ml}$ is the usual target level.

Platelets are given either as pools prepared from five to eight random-donor platelets or as a single SDAP. In an unsensitized patient without increased platelet consumption, six to eight units of RD platelets (about 1 unit per $10 \mathrm{Kg}$ body weight) are transfused and each unit is anticipated to increase the platelet count by 5000 to $10000 /$ $\mathrm{mL}$. Patients receiving multiple transfusions usually get alloimmunized to many HLA and platelet specific antigens and have little or no increase in their post transfusion platelet counts. Patients who may require multiple transfusions are best served by SDAP and leukocyte reduced components to reduce their risk of alloimmunization. Refractoriness to 
multiple transfusions may be evaluated by using corrected count increment $(\mathrm{CCl})$ :

$\mathrm{CCl}=$ [posttransfusion count-pretransfusion count/number of platelets transfused $\times 10^{11}$ ] x (Body surface area in square metres)

The platelet count performed one hour after the transfusion is acceptable if the $\mathrm{CCl}$ is $10 \times 10^{9} / \mathrm{ml}$, and after $18-24$ hours an increment of $7.5 \times 10^{9} / \mathrm{ml}$ is expected. Patients who have suboptimal responses are likely to have received multiple transfusions and have antibodies directed against Class 1 HLA antigens. Refractoriness can be detected by anti-HLA antibodies in the recipient's serum. These patients are best served by SDAP if needed.

Fresh Frozen Plasma : FFP contains components of the coagulation, fibrinolytic, and complement systems. It is obtained from single donor. It is frozen at $8^{\circ} \mathrm{C}$, this temperature protects Factor V and VII mainly. It carries the same risk of HIV and Hepatitis as PRBC. It is useful in treating deficiencies in 2, 5, 7, 8, 9, 10, 11, also in Coumarin reversal, antithrombin III deficiency. Type and Rh specific plasma should be used. Urticaria and fatal pulmonary edema can occur. Plasma may also be collected by apheresis.

Plasma derivatives such as albumin, intravenous immunoglobulin, antithrombin, and coagulation factors are prepared from pooled plasma from many donors and are treated to eliminate infectious agents.

Cryoprecipitate :

It is used to replenish Factor VIII or fibrinogen. It is formed as a plasma concentrate that consists primarily Factor VIII and fibrinogen. In addition, it also contains Factor XIII, vWF and fibronectin. It is stored at $37^{\circ} \mathrm{C}$ since higher temperatures destroy Factor VIII. The main disadvantage is the increased risk of hemolytic reactions due to small amounts of anti-A, anti-B, and $\mathrm{Rh}$ antibodies left over in preparation as it is obtained from multiple donors.

\section{Alternatives to Transfusion}

Autotransfusion: It involves collection and immediate reinfusion of patient's own blood for volume replacement and to increase red cell mass. Massive exsanguinations from either blunt or penetrating trauma without gross enteric contamination are best candidates. It eliminates risk of histocompatibility reactions and transfusion transmitted infections. The most common complication is thrombocytopenia. When patients receive more than $4 \mathrm{~L}$ of blood, platelet count may drop to less than $50,000 / \mathrm{mL}$, and risk of acute tubular necrosis increased from debris of plasma-free $\mathrm{Hb}$. There also exist increased risks of air embolism, particulate microemboli, and disseminated intravascular coagulation(DIC).

Pre-Autologous blood storage: It is similar to PRBC (42 days maximum). Contraindications include significant coronary artery disease, chronic obstructive pulmonary disease, and existence of a hematologic disorder. This is not recommended in pregnancy.

Oxygen carrying blood substitutes: i.e. perfluorocarbons and aggregated hemoglobin solutions are presently in various stages of trials.

Intraoperative cell salvage (IOCS) is the process by which blood shed within the surgical field is retrieved by an anticoagulated suction apparatus and collected within a reservoir from where it is centrifuged, washed and pumped into an infusion bag. This salvaged blood can then be returned to the patient. This process is effective in reducing the need for allogenic red cell transfusion and has been widely used in adult orthopaedic and cardiac surgeries without complications. Cell salvage is recommended for women in whom an intraoperative blood loss of more than $1500 \mathrm{ml}$ is anticipated.

The use of IOCS in obstetric practice has been limited, owing to concerns about contamination by amniotic fluid, specifically the risks of amniotic fluid embolism, and by fetal blood cells, particularly the risk of anti-D formation. Common markers of amniotic fluid contamination can be found in the maternal circulation after most deliveries. The IOCS, together with the use of modern leucocyte depletion filters, has been shown to be effective at removing the common markers of amniotic fluid embolism. ${ }^{5}$ However, it will not remove fetal blood cells and therefore adequate anti-D immunisation (as determined by Kleihauer test one hour after the procedure) will be required to prevent rhesus immunisation in Rh D-negative women. ${ }^{6}$ IOCS has been used during caesarean section in a number of case series and trials without any reported complications related to receiving salvaged blood. ${ }^{7}$

The National Institute for Health and Clinical Excellence NICE guideline on intraoperative blood cell salvage in obstetrics (IOCS) in obstetrics recommends that, when used, it should be used by healthcare teams who use it regularly and have built up expertise and experience, that patients should consent to its use, and this should be subject to audit and monitoring. ${ }^{7}$

\section{Blood Transfusion in Major Obstetrics Hemorrhage}

Despite the risks associated with transfusions, obstetricians are frequently too aggressive in transfusing blood and blood products to their patients. Acute blood loss in obstetrics is usually due to placenta praevia, postpartum blood loss and surgery related. This demands early involvement of a consultant obstetrician, anaesthetist, haematologist and the blood bank.

There are no established criteria for initiating red cell transfusions. Decision should be based on clinical and haematological parameters.

- Transfusion is advocated when the PCV is less than $21 \%$ (in patients without any cardiac pathology) and is generally infused in a ratio of 3 volumes of crystalloid to 1 volume of blood. In severe shock this ratio may increase up to 8:1. 
- Infusion of FFP should be considered before one blood volume is lost.

- Fibrinogen deficiency is the earliest haemostatic abnormality encountered when packed red cell concentrates are used to replace major blood loss. Clinically significant fibrinogen deficiency develops after a loss of about $150 \%$ of blood volume.

- The platelet count should not be allowed to fall below $50 \times 10^{9} / \mathrm{mL}$ in the acutely bleeding patient. A platelet transfusion trigger of $75 \times 10^{9} / \mathrm{mL}$ is recommended to provide a margin of safety. A platelet count of $50 \times 10^{9} / \mathrm{mL}$ may be anticipated when approximately two blood volumes have been replaced by fluid or red cell components.

- Anti-Rh D (250 IU) will be needed if the platelets are Rh $D$ positive and the recipient $R h D$ negative. This is not necessary if a caesarean hysterectomy has been performed.

- The FFP and cryoprecipitate should ideally be of the same group as the recipient. If unavailable, FFP of a different $A B O$ group is acceptable, provided that it does not have a high titre anti-A or anti-B activity. ${ }^{9}$ No anti-D prophylaxis is required if an $R h D$ negative woman receives $R h D$ positive FFP or cryoprecipitate.

- In the bleeding woman with DIC, a combination of FFP, platelets and cryoprecipitate is indicated.

- Fibrinogen levels should be maintained above $1.0 \mathrm{~g} / \mathrm{L}$ by the use of FFP. ${ }^{10}$

- Dilution of coagulation factors is the primary cause of coagulopathy in major blood loss following volume replacement with crystalloid or colloid and transfusion of red cell components.

- Women who have been exposed to prolonged hypoxia, hypovolaemia or hypothermia (for instance, owing to inadequate resuscitation), amniotic fluid embolism, placental abruption and pre-eclampsia are at risk of DIC. If DIC is strongly suspected in these women and clotting studies take a long time, transfusion of FFP should be considered before results are available if hemorrhage is otherwise difficult to control.

- DIC should be suspected when there is profuse bleeding from the site of trauma and oozing from the sites of venepuncture and intravenous line insertions. There are no data to substantiate transfusion triggers for clotting factors but common practice is to administer FFP $12-15 \mathrm{~mL} / \mathrm{kg}$ to keep the activated partial thromboplastin time (aPTT) and prothrombin time ratios less than 1:5.

- The use of rFVIla (recombinant factor VIla) may be considered as a treatment for life-threatening postpartum haemorrhage but it is not a substitute for life-saving procedures like embolisation or surgery; which need to be performed promptly or alternatively, the patient needs to be transferred to a referral centre for the same.

- Blood transfusion of banked blood results in provoking neutrophil cytotoxicity which is now regarded as a key mechanism in multiple organ failure (MOF) and early transfusion has been shown to be a strong independent risk factor for MOF. ${ }^{11}$ Thus in acute blood loss, with moderate haemorrhagic shock, either normal saline or Ringer's lactate may be used for volume replacement and in massive hemorrhagic shock, Ringer's lactate may be the fluid of choice. ${ }^{12}$ Colloids have been largely replaced by crystalloids due to the propensity of the former to leak from permeable capillary membranes thereby worsening oedema and impairing tissue oxygenation. However, large volumes of crystalloids have been implicated in the etiology of adult respiratory distress syndrome and the abdominal compartment syndrome, which colloids may not cause, but overall crystalloids provide the best survival. ${ }^{13}$

\section{General Principles of Blood Transfusion in Obstetric Patient}

- Red cell alloimmunisation is most likely to occur in the last trimester, therefore no pre-transfusion sample should be more than seven days old and ideally should be freshly drawn. ${ }^{14}$

- Only Kell-negative blood should be used for transfusion in women of childbearing age, owing to the high risk of alloimmunisation and subsequent hemolytic disease of the newborn (unless a woman is known to be Kell positive).

-In pregnancy, pre-autologous deposit is not recommended.

- Cell salvage should only be used by healthcare teams who use it regularly and have the necessary expertise and experience.

- If the $\mathrm{Hb}$ is less than $7 \mathrm{~g} / \mathrm{dL}$ in labour or in the immediate postpartum period, the decision to transfuse should be made according to the individual's medical history, age and symptoms.

- If the $\mathrm{Hb}$ is less than 7-8 $\mathrm{g} / \mathrm{dL}$ in postnatal period, where there is no continuing or threat of bleeding, the decision to transfuse should be made on an informed individual basis. In fit, healthy, asymptomatic patients there is little evidence of the benefit of blood transfusion.

- If unexpected severe bleeding is encountered during delivery, investigations should be made post-natally to rule out a bleeding diatheses. These investigations should be performed on a non-urgent basis at least 3-6 months after delivery.

\section{Practical guidelines}

- PCV may be converted to Haemoglobin in $\mathrm{gm} / \mathrm{dL}$ by dividing it by 0.03 .

- One unit of blood lost is compensated by 3 units of electrolytes. A blood loss of $1500 \mathrm{~mL}$ is thus compensated by $1500 \times 3=4500 \mathrm{~mL}$ Ringer or normal saline infusion.

- In serious hypovolemia, volume therapy with Ringer lactate or normal saline infusion is immediately started. If her circulatory state does not stabilize prompt and 
permanently as a response to $3000 \mathrm{ml}$ infusion, blood transfusion (or plasma expander) is indicated.

- Once the FFP has been ordered, the blood bank takes at least 30 minutes to thaw and issue the same. During this time, resuscitation should be continued with volume

\section{REFERENCES}

1. Serious Hazards of Transfusion Steering Group. Serious Hazards of Transfusion (SHOT) Annual Report 2004. London: Royal College of Pathologists; 2004.

2. Practice strategies for elective red blood cell transfusion. American College of Physicians. Ann Intern Med. 1992;116:403-406.

3. Innes G. Guidelines for red blood cells and plasma transfusion for adults and childred: an emergency physician's overview of the 1997 Canadian blood transfusion guidelines. Part 1: red blood cell transfusion.Canadian Medical Association Expert Working Group. J Emerg Med. 1998;16:129-131.

4. Dzieczkowski JS, Anderson KC. Transfusion Biology And Therapy. In: Kasper DL, Fausi AS, Longo DL, Braunwald E, Hauser SL, Jameson JL (eds). Harrison's Principles of Internal Medicine. 17th edition. McGraw Hill: New York; 662-67.

5. Waters JH, Biscotti MD, Potter PS, Phillipson E. Amniotic fluid removal during cell salvaged in the caesarean section patient. Anesthesiology 2000;92:1531-6.

6. Catling SJ, Williams S, Fielding AM. Cell salvage in obstetrics: an evaluation of the ability of cell salvage combined with leucocyte depletion filtration to remove amniotic fluid from operative blood loss at caesarean section. Int J Obstet Anesth. 1999;8:79-84.

7. National Institute for Health and Clinical Excellence. Intraoperative Blood Cell Salvage in Obstetrics. Interventional Procedure Guidance 144. London: NICE; 2005. expanding fluids or red cells as appropriate.

- Platelets may be transfused via an unused blood-giving set, although a platelet-giving set reduces wastage because it has less dead space. Transfusion of platelets through a transfusion set previously used for red cells is not recommended.

8. British Committee for Standards in Haematology, Blood Transfusion Task Force. Guidelines for the Clinical Use of Red Cell Transfusions. Br J Haematol. 2001;113:24-31.

9. British Committee for Standards in Haematology Blood Transfusion Task Force. Guidelines for the use of FFP, cryoprecipitate and cryosupernatant. Br J Haematol. 2004;126:11-28.

10. Stainsby D, MacLennan S, Thomas D, Isaac J, Hamilton PJ. British Committee for Standards in Haematology. Guidelines on the managementof massive blood loss. Br J Haematol. 2006;135:634-41.

11. Botha AJ, Moore FA, Moore EE. Postinjury neutrophil priming and activation: an early vulnerable window. Surgery. 1995;118:358-365.

12. Healy MA, Davis RE, Liu FC. Lactated Ringer's is superior to norma saline in a model of massive hemorrhage and resuscitation. J trauma 1998; 45:894-899.

13. Moore FA, Mckinley BA, Moore EE. The next generation in shock resuscitation. Lancet. 2004;363:1988-1996.

14. British Committee for Standards in Haematology. Blood Transfusion Task Force. Guidelines for compatibility procedures in blood transfusion laboratories. Working Party of the British Committee for Standards in Haematology. Blood Transfusion Task Force. Transfusion Medicine. 2004;14:59-73. 